\title{
Atomic force microscope with integrated optical microscope for biological applications
}

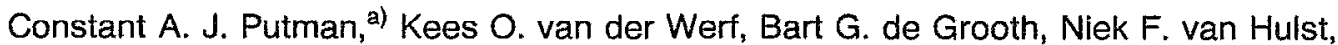 \\ Frans B. Segerink, and Jan Greve \\ University of Twente, Department of Applied Physics. P.O. Box 217. 7500 AE Enschede, The Netherlands
}

(Received 26 September 1991; accepted for publication 4 November 1991)

\begin{abstract}
Since atomic force microscopy (AFM) is capable of imaging nonconducting surfaces, the technique holds great promises for high-resolution imaging of biological specimens.

A disadvantage of most AFMs is the fact that the relatively large sample surface has to be scanned multiple times to pinpoint a specific biological object of interest. Here an

AFM is presented which has an incorporated inverted optical microscope. The optical image from the optical microscope is not obscured by the cantilever. Using a $X Y$ stage to move the sample, an object is selected with the optical microscope and an AFM image of the selected object can be obtained. AFM images of chromosomes and K562 cells show the potential of the microscope. The microscope further enables a direct comparison between optically observed features and topological information obtained from AFM images.
\end{abstract}

\section{INTRODUCTION}

With the introduction of the atomic force microscope (AFM), ${ }^{1}$ a powerful tool has become available for highresolution imaging of biological objects with dimensions ranging from DNA molecules ${ }^{2}$ to blood cells. ${ }^{3}$ Unlike other techniques with similar resolution, such as scanning tunneling microscopy (STM) and scanning electron microscopy (SEM), AFM requires no coating of the specimen. The demonstration by Hansma and co-workers that the AFM can be operated in an aqueous environment ${ }^{4}$ holds great promises for biological studies under physiological conditions. In practice, most "imaging time" is used to locate specific objects of interest, which are more or less randomly distributed on the sample substrate, by making multiple AFM images. For objects with the size of $1 \mu \mathrm{m}$ or larger, we have solved this problem by constructing an AFM which is integrated in an inverted optical microscope (EPI illumination). With this instrument an object of interest can be selected quickly and a direct comparison between the optical image (in reflection or in fluorescence) and the AFM image can be made due to the overlapping field of view.

\section{INSTRUMENT DESIGN}

A problem encountered in integrating an optical microscope in an AFM is that the tip and the cantilever are directly located on top of the object. In some commercially available $\mathrm{AFMs}^{5,6}$ special long-working-distance objectives are used to obtain an optical image from the top. In that configuration, part of the sample is hidden from direct optical viewing. Also other designs incorporating an optical microscope have this problem. ${ }^{7-10}$

Since most biological objects are transparent, an optical image can be obtained from the back side by using a

\footnotetext{
a) To whom correspondence should be directed.
}

transparent object carrier, e.g., a microscope slide. For this purpose we have taken a small objective lens from a compact disk player (outer diameter $8 \mathrm{~mm}$, Philips, The Netherlands) which is positioned inside the hollow piezo tube (inner diameter $16 \mathrm{~mm}$, Staveley Sensors, East Hartford, CT) used for scanning the sample in the $X-Y-Z$ direction (Fig. 1). The objective can be adjusted by $X Y-2$ (range: $2 \times 2 \mathrm{~mm}^{2}$ ) such that the objective and the AFM tip are positioned on one line. By rotating the long tube on which the lens is placed inside the $X Y-2$ stage, the lens can be focused and an optical image of the AFM tip is obtained. The illumination consists of a mercury are lamp (100 W, Osram, Germany) and a dichroic filter block (Nikon). The dichroic filter block consist of a dichroic mirror with $50 \%$ transmittance at $580 \mathrm{~nm}$, an excitation filter that selects the green line at $546 \mathrm{~nm}$ from the spectrum and an optional long-wave-pass filter with a cut-on wavelength of $590 \mathrm{~nm}$. If no long-wave-pass filter is used, the optical image is a reflection image. If it is used, a fluorescence image is obtained. The optical image is detected by a CCD camera (WVF10, Panasonic) and displayed on a video monitor (C-S2181ET, JVC). In reflection a slightly better image is obtained when two crossed polaroids (one in the illumination path, the other in the detection path) and a quarter-wave plate (between lens and sample) are used to eliminate any stray light that is not originating from the sample.

The hollow piezo tube, on top of which the sample (on a microscope slide, in air, or in a cuvet under a fluid) is placed, can be moved with respect to both the tip and the objective using a $X Y$-translation stage $(X Y-1)$. This $X Y$ stage consists of two interconnected translation stages carrying a large bore ( $35 \mathrm{~mm}$ diam, Micro-Controle, France). The $X Y$ stage is attached upside down to the base plate. In order to prevent contact between piezo tube and lens tube, small blocks are positioned inside the $X Y$ stage to restrict the travel range of the stage. In this way an object field with a size of $3 \times 3 \mathrm{~mm}^{2}$ can be screened with the optical 


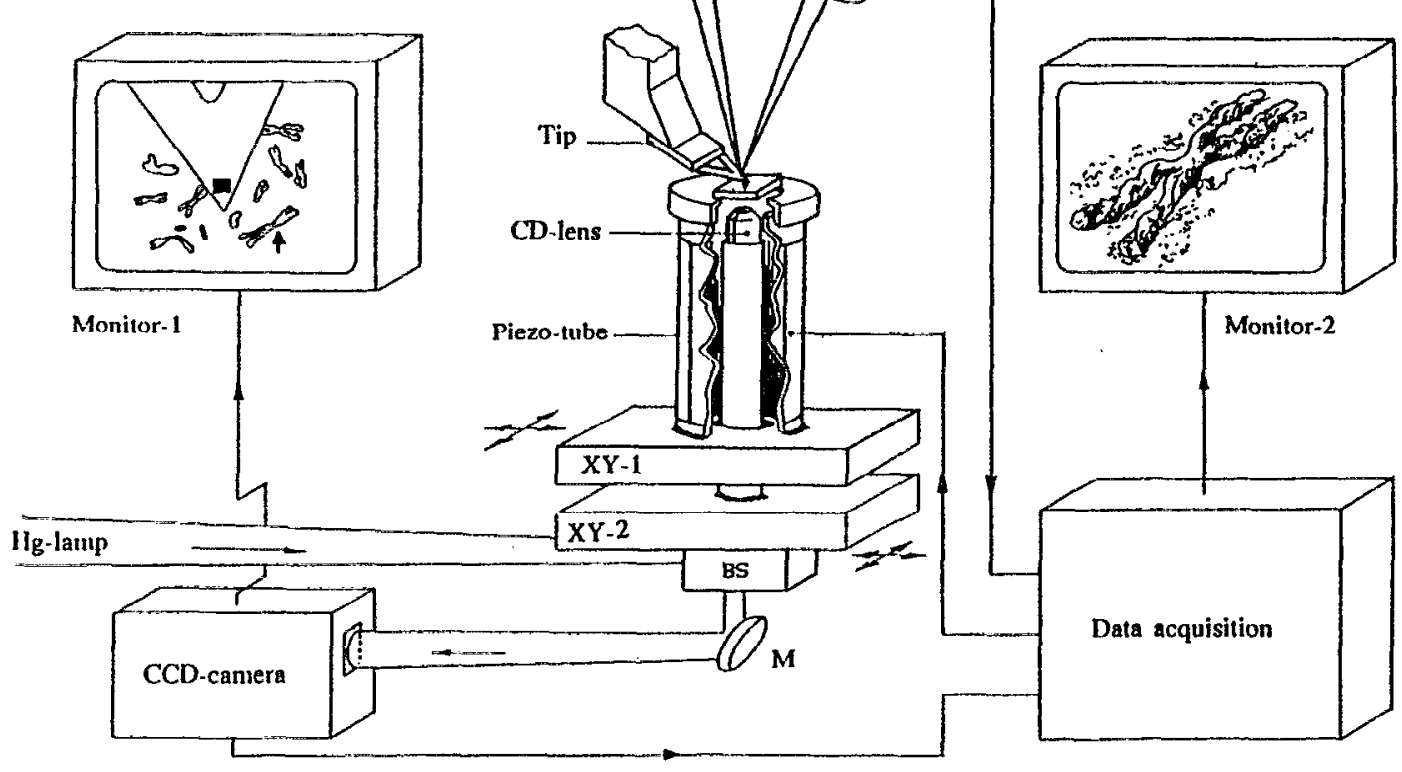

FIG. 1. Schematic representation of the AFM integrated with an optical microscope. The optical microscope consists of a mercury arc lamp for illumination, a dichroic beam splitter (BS), a beam steering mirror (M), a CD lens as an objective and CCD camera with video monitor (monitor 1 ). The CD lens is located on top of a long hollow tube and can be moved by $X Y-2$. The CD lens can be translated in the $Z$ direction in order to focus the sample (details not shown). The AFM consists of a piezo tube for sample scanning and a commercial, V-shaped $\mathrm{Si}_{3} \mathrm{~N}_{4}$ cantilever (200 $\mu \mathrm{m}$ long) with an integrated tip. The displacement of the cantilever is measured by the optical beam deflection method using a diode laser and a quadrant detector. The data acquisition is based on a HP9000-330 work station and a VME-bus system. The AFM images are displayed on monitor 2 . The sample can be moved over a range of $3 \times 3 \mathrm{~mm}^{2}$ by $X Y-1$.

microscope. The numerical aperture of the lens is 0.4 , which results in an optical resolution of about $1 \mu \mathrm{m}$. The field of view is $100 \mu \mathrm{m}$ in diameter. The sample level is 2 $\mathrm{mm}$ above the base plate on which an optical beam deflection $^{11}$ module for cantilever displacement detection is positioned. The module consists of a laser diode $(1 \mathrm{~mW}$, $780 \mathrm{~nm}$ ) and a collimating lens both taken from the same compact disk player mentioned before. An $80-\mathrm{mm}$ lens focuses the collimated beam at the gold-coated cantilever. ${ }^{5}$ Since the reflected beam is detected by a quadrant detector (C30845, RCA), we are capable of measuring the friction and the deflection of the cantilever simultaneously. In order to prevent interference with the optical image in the fluorescence mode, a 780-nm laser diode and not a red 670-nm laser diode is used to detect the cantilever displacement. In front of the CCD camera an additional shortwave-pass filter (cutoff wavelength at $660 \mathrm{~nm}$, Ealing Electro-Optics Inc., South Natick, MA) is positioned to block the light from the laser diode (not shown in Fig. 1).

After selecting an object of interest from the optical image, the object is placed under the tip and an AFM image is obtained. The lateral resolution is a few nanometers and the noise level in a $10-\mathrm{kHz}$ bandwidth corresponds to a cantilever displacement of $1 \AA$. This noise level is primarily due to electronic noise and mechanical and acoustic vibrations (no extensive vibration isolation precautions have been taken yet). The maximum scan range is $15 \times 15 \mu \mathrm{m}^{2}$. The data acquisition is based on an HP9000330 work station (Hewlett Packard) and a VME-bus system containing an HP interface, a framegrabber (VFG100,
Imaging Technology Inc, Woburn, MA), AD converters, and a scan generator. The high-voltage amplifiers (range -300 to $+300 \mathrm{~V})$ for the piezo tube, the control electronics, and the power supply for the laser diode are home built. An image of $512 \times 512$ pixels takes between $15 \mathrm{~s}$ (fast scan) and 2 min (slow scan), and they are displayed on a monitor (Multisync 2, NEC). In this report only the deflection mode and not the friction mode is used. The AFM images presented here are obtained in the constant force mode.

\section{RESULTS AND DISCUSSION}

In Fig. 2 the optical image (in reflection) of a sample of metaphase chromosomes is shown. In this picture the V-shaped microfabricated cantilever $\left(\mathrm{Si}_{3} \mathrm{~N}_{4}\right)^{12}$ is clearly observed, with the square area of $4 \times 4 \mu \mathrm{m}^{2}$ in the middle of which the tip with a radius of typically $30 \mathrm{~nm}$ is located. Notice the beautiful interference pattern (Newton fringes) originating from the interference between light reflected from the sample carrier surface and the tilted cantilever. With the relatively monochromatic green light used for illumination $(546 \mathrm{~nm})$, the observed spacing between the fringes is in agreement with a tilt angle between the cantilever and the substrate of $15^{\circ}$. The white spot is from the laser beam sensing the displacement of the cantilever.

In Fig. 3 AFM images at low and high resolution are shown of a metaphase chromosome that was selected from the optical image. This example demonstrates the possibilities of the instrument. The high resolution of the AFM 


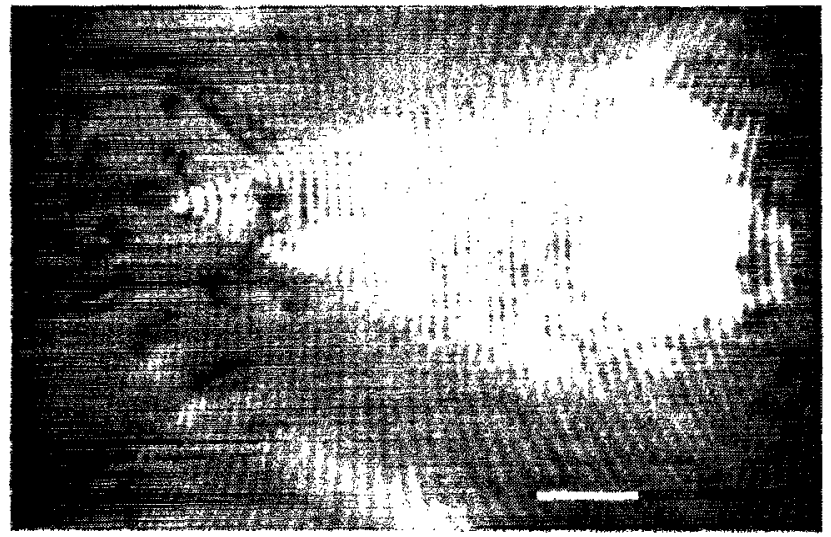

FIG. 2. Optical image in reflection of a sample of metaphase chromosomes taken from a Chinese hamster lung cell line (CHL-V79), prepared according to method described elsewhere (see Ref. 18). Bar: $10 \mu \mathrm{m}$.

reveals fine details in the chromosome structure which up to now are only visible with coated samples using SEM. In Fig. 3(a) we see that the chromatids display a pattern of loops with a period of about $800 \mathrm{~nm}$. This confirms earlier observations on coiling of the chromatids. ${ }^{13,14}$ In images with a higher resolution, structures with sizes in the range of 50-60 nm are predominant [Fig. 3(b)]. Similar struc-

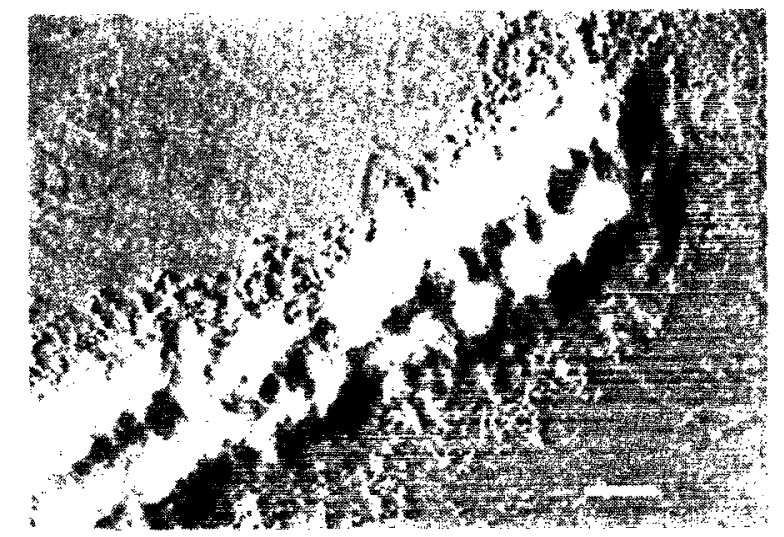

(a)

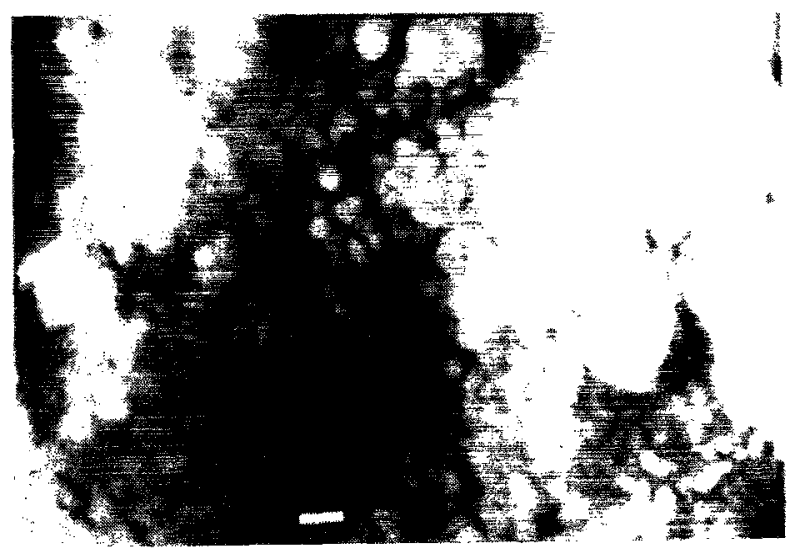

(b)

FIG. 3. AFM images of a single CHL metaphase chromosome. The applied force is $5 \mathrm{nN}$. (a) Bar: $1 \mu \mathrm{m}$; (b) bar: $100 \mathrm{~nm}$.

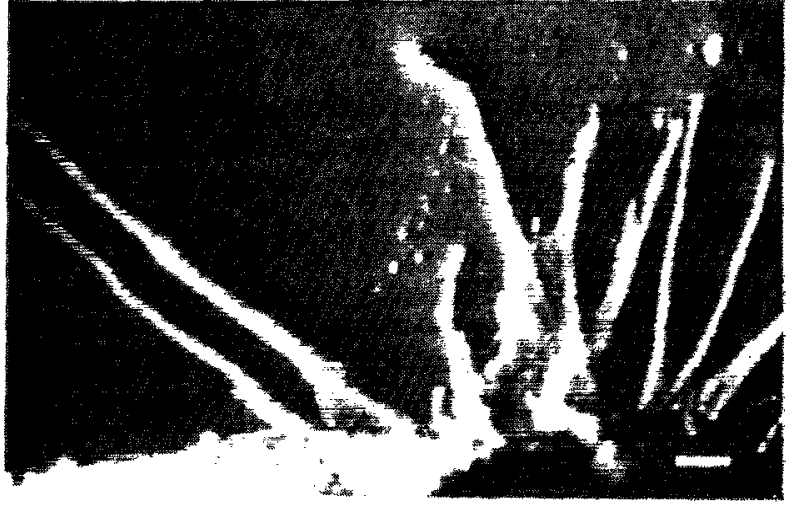

(a)
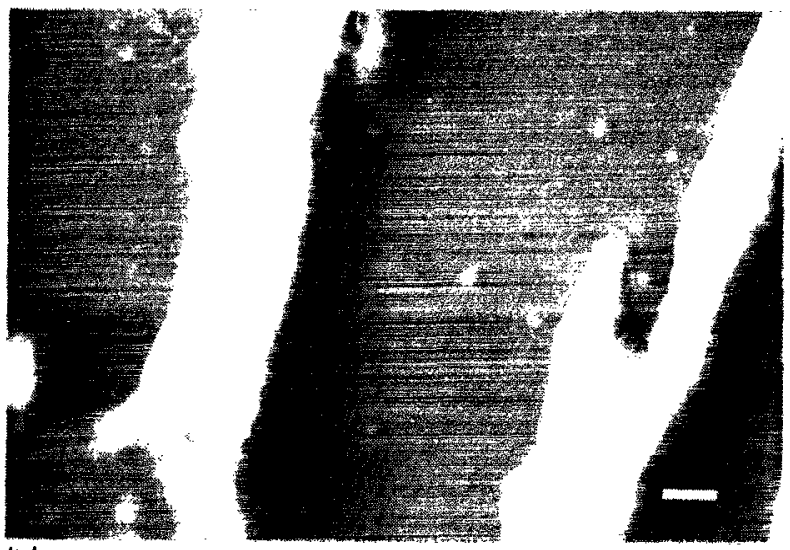

(b)

FIG. 4. AFM images of a $K 562$ cell. $\mathrm{K} 562$ cells are incubated at $37^{\circ} \mathrm{C}$ for $30 \mathrm{~min}$ in RPMI buffer on a poly-L-lysine-coated microscope slide. The slide is dipped in a PEM buffer, $p \mathrm{H} 6.5$, at $37^{\circ} \mathrm{C}$. Then the cells are fixated for $30 \mathrm{~min}$ at room temperature. Finally, the cells are dehydrated and desalinated by washing in cold, pure alcohol and are allowed to air dry. The applied force is $5 \mathrm{nN}$. (a) Bar: $1 \mu \mathrm{m}$; (b) bar: $250 \mathrm{~nm}$.

tures have been visualized by SEM and have been interpreted as to present the ends of loops of $30-\mathrm{nm}$ chromatin fibers, ${ }^{15}$ where the fiber reaches the surface and folds back into the body of the chromosome. ${ }^{16}$ The fine details observed at the boundaries of the chromatids [Fig. 3(a)] have the same size as the structures in Fig. 3(b) and seem to originate from the chromosome.

As a second example, AFM images of a cell from a human cell line (K562, predecessor of erythroblasts) are shown in Fig. 4. The K562 cells are allowed to adhere on a poly-L-lysine-coated microscope slide. After $30 \mathrm{~min}$ adhesion structures are observed. These spikelike filopodia have a width of $200-400 \mathrm{~nm}$ and have an average height of $50 \mathrm{~nm}$. On the filopodia transverse grooves at irregular distances of 100-200 $\mathrm{nm}$ are clearly visible [see, for instance, the two filopodia on the left-hand side of Fig. 4(a)]. In Fig. 4(b) even smaller structures are observed.

It is also possible to compare optically observed features and topological information obtained from the AFM images. For instance, the instrument enables direct comparison between the optically observed banding patterns after treatment and the topology of the chromosomes (pre- 
liminary results). Currently we are studying the chromosome appearance under different physiological conditions, such as the influence of divalent cations, ${ }^{17}$ in order to obtain a better understanding of the chromosome structure.

\section{CONCLUSION}

The atomic force microscope with an integrated optical microscope as presented here is an instrument that is easy to operate and can be a useful tool for biologists to study biological structures and processes on a molecular scale under physiological conditions. Using an $X Y$ stage to move the sample and the optical microscope to pinpoint an object of interest, the imaging time is reduced significantly. The instruments cover an area of $3 \times 3 \mathrm{~mm}^{2}$ with a lateral resolution of a few nanometers. Optically observed features and topological information obtained from AFM images can be compared and can give additional information for biological studies.

\section{ACKNOWLEDGMENTS}

The authors thank K. Smit for machining the mechanical components, and G. M. J. Segers and Y. M. Kraan for sample preparation. This research was supported by the Netherlands Organization of Scientific Research (NWO).
'G. Binnig, C. F. Quate, and Ch. Gerber, Phys: Rev. Lett. 56, 930 (1986).

${ }^{2}$ S. M. Lindsay, L. A. Nagahara, T. Thundat, U Knipping, R. L. Rill, B. Drake, C. B. Prater, A. L. Weisenhorn, S. A. C. Gould, and P. K. Hansma, J. Biomed. Struct. Dyn. 7, 279 (1989).

${ }^{3}$ S. A. C. Gould, B. Drake, C. B. Prater, A. L. Weisenhorn, S. Manne, H. G. Hansma, P. K. Hansma, J. Massie, M. Longmire, V. Elings, B. Dixon Northern, B. Mukergee, C. M. Peterson, W. Stoeckenius, T. R. Albrecht, and C. F. Quate, J. Vac. Sci. Technol. A 8, 369 (1990).

${ }^{4}$ B. Drake, C. B. Prater, A. L. Weisenhorn, S. A. C. Gould, T. R. Albrecht, C. F. Quate, D. S. Cannell, H. G. Hansma, and P. K. Hansma, Science 243, 1586 (1989).

${ }_{5}^{5}$ Park Scientific Instruments, Sunnyvale, CA.

${ }^{5}$ Digital Instruments, Santa Barbara, CA.

${ }^{7}$ W. Wiegräbe, H. Breu, R. Gatz, K. Levai, and R. Guckenberger, U1tramicroscopy (to be published).

${ }^{8}$ R. Kaneko, R. Matsuda, S. Oguchi, S. Hara, T. Okada, H. Ogawa, and Y. Nakamura, Ultramicroscopy (to be published).

${ }^{9}$ M. Radmacher, B. Goettgens, R. Tillman, and H. E. Gaub, Ultramicroscopy (to be published).

${ }^{10}$ W. Häberle, J. K. H. Hörber, and G. Binnig, J. Vac. Sci. Technol. B 9. 1210 (1991).

${ }^{11}$ G. Meyer and N. M. Amer, Appl. Phys. Lett. 53, 2400 (1988).

${ }^{12}$ T. R. Albrecht, S. Akamine, T. E. Carver, and C. F. Quate, J. Vac. Sci. Technol. A 8, 3386 (1990).

${ }^{13}$ E. Boy de la Tour and U. K. Laenmli, Cell 55, 937 (1988).

${ }^{14}$ A. L. Bak, J. Zeuthen, and F. H. C. Crick, Proc. Natl. Acad. Sci. USA 74, 1595 (1977).

${ }^{15}$ E. J. Dupraw, Nature 206, 338 (1965).

${ }^{16}$ C. J. Harrison, M. Britch, T. D. Allen, and R. Harris, Exp. Cell. Res. 134, 141 (1981).

${ }^{17}$ K. W. Adolph and L. R. Kreisman, Exp. Cell Res. 147, 155 (1983).

${ }^{18}$ J. C. K. Lee and G. F. Bahr, Chromosoma 88, 374 (1983). 